\title{
Constrained Genetic Disentangling of Close Binary Spectra
}

\author{
V. Kolbas ${ }^{1}$ and K. Pavlovski ${ }^{1,2}$ \\ ${ }^{1}$ Department of Physics, University of Zagreb, 10000 Zagreb, Croatia \\ ${ }^{2}$ Astrophysics Group, EPSAM, Keele University, Staffordshire, ST5 5BG, UK
}

\begin{abstract}
The spectral disentangling technique makes possible separation of individual component spectra in binary or multiple systems, and determination of the orbital elements in a self-consistent way. Since its introduction, a number of variants of their basic idea have been implemented. We present yet another 'direct' approach using optimization by genetic algorithm. Starting with an initial random flux distribution representing individual spectra, genetic optimization returns both individual component spectra and an optimal set of orbital parameters only constrained by time-series of the observed composite spectra of the binary system. Benchmark tests on V453 Cyg, which is an eclipsing binary with total eclipse, as well as tests on the artificial time-series spectra, have proven that 'constrained genetic disentangling' is performing correctly and efficiently, albeit with high demand on CPU time. Since genetic optimization can be easily parallelized, we expect our second release to run on cluster in a less time-consuming way.
\end{abstract}

Keywords. genetic algorithm, spectral disentangling

\section{Introduction}

The spectral disentangling technique, as invented by Simon \& Sturm (1994) and Hadrava (1995), makes possible separation of individual component spectra in binary or multiple systems, and determination of orbital elements in a self-consistent way. One only needs a time series of binary star spectra. There is no need for template spectra as in the technique of cross-correlation, since they are the main source of bias in measuring RVs of the components, and hence determination of orbital elements (see detailed discussion in the review paper by Hensberge \& Pavlovski 2007). In spectral disentangling (SPD, here-in-after), the role of templates is overtaken by the spectra of components themselves. The advantage of SPD is obvious, besides the set of orbital elements, individual spectra of the components are calculated. These separated spectra of the components then can be analyzed by all means as single star spectra and a variety of important astrophysical informations can be extracted (c.f. Pavlovski \& Hensberge 2010, Pavlovski \& Southworth 2011, these proceedings).

A number of different techniques have been implemented to separate individual spectra of components from complex binary (or multiple) star spectra. They go from a simple subtraction technique to very sophisticated numerical methods. The reader is referred to review papers by Hadrava $(2004,2009)$ and Pavlovski \& Hensberge (2010) where details on these techniques can be found, as well as references to original works (c.f. Hadrava, these proceedings).

\section{Genetic forward disentangling}

SPD in the formulation of Simon \& Sturm (1994) is based on solving the matrix equation $\mathrm{Ax}=\mathrm{y}$, where vector $\mathrm{y}$ contains all observed spectra, and vector $\mathrm{x}$ contains the spectra 
of the components. Matrix A has elements (blocks) corresponding to Doppler shifts and light dilution factors. The system is an over-determined system of linear equations (more equations than unknowns) since usually in practice we have more observed spectra than stellar components. Therefore, a least squares solution is required in order to minimize the norm of the residuals $r=\|A x-y\|$. On the computational side, this method is very demanding. Fourier disentangling as invented by Hadrava (1995) overcomes this problem. The method is not limited by the number of input spectra or the length of the spectral interval. The only limitation is that both ends of the spectral range to be disentangled should be exactly on the continuum.

A binary star spectrum is a linear combination of two (or more, for a multiple system) individual spectra of components which are shifted for appropriate RVs in a given phase of the orbital cycle. Therefore, we can perform direct disentangling seeking for two spectra which, for a given set of orbital phases, would be constrained by time series of observed spectra. Like in the SPD method in velocity space of Simon \& Sturm (1994), we are seeking a least square solution and minimize squared residuals. In this direct disentangling technique, we have used optimization by genetic algorithm (Charbeonneau 1995).

We have performed a number of different tests on simulated and real data. Special attention has been given to reproduce disentangled component spectra in the benchmark binary star V453 Cyg (Simon \& Sturm 1994, Pavlovski \& Southworth 2009) which has a total eclipse, and the spectrum of one of the components can be directly observed. An important aspect of genetic constrained disentangling is the excellent performance in disentangling difficult cases like broad Balmer lines or, even more significant, disentangling a spectral range that is cut in the line wing(s). This is a severe problem for Fourier disentangling since weights can be assigned only to spectra, and not to pixels. With growing numbers of faint binaries observed spectroscopically in our galaxy, or in galaxies of the Local Group (North et al. 2010), which contain nebular emission in Balmer lines, and/or strong interstellar bands, these fine characteristics are of particular importance. Now, we are working on improving the performance of the genetic algorithm code since CPU time is not yet competitive with other methods.

\section{References}

Charbonneau, P. 1995, ApJS, 101, 309

Hadrava, P. 1995, A\&AS, 114, 393

Hadrava, P. 2004, ASP-CS, 318, 86

Hadrava, P. 2009, arXiv:0909.0172

Hensberge, H. \& Pavlovski, K. 2007, in IAU Symp., 240, 136

North, P., Gauderon, R., Barblan, F., \& Royer, F., 2010 A\&A, 520, A74

Pavlovski, K. \& Hensberge, H. 2010, ASP-CS, 435, 207

Pavlovski, K. \& Southworth, J. 2009, MNRAS, 394, 1519

Simon, K. P. \& Sturm, E., 1994, A\&A, 281, 286 